\title{
Fine Mapping and Identification of BnaC06.FtsH1, a Lethal Gene That Regulates the PSII Repair Cycle in Brassica napus
}

\author{
Kai Xu, Yujin Wu, Jurong Song, Kaining Hu D , Zengxiang Wu, Jing Wen, Bin Yi, Chaozhi Ma, Jinxiong Shen, \\ Tingdong $\mathrm{Fu}$ and Jinxing $\mathrm{Tu}$ * (D)
}

check for updates

Citation: $\mathrm{Xu}, \mathrm{K} . ; \mathrm{Wu}, \mathrm{Y}$;; Song, J.; Hu, K.; Wu, Z.; Wen, J.; Yi, B.; Ma, C.;

Shen, J.; Fu, T.; et al. Fine Mapping and Identification of BnaC06.FtsH1, a Lethal Gene That Regulates the PSII Repair Cycle in Brassica napus. Int. J. Mol. Sci. 2021, 22, 2087. https:// doi.org/10.3390/ijms22042087

Academic Editor: Atle M. Bones Received: 14 January 2021 Accepted: 16 February 2021 Published: 19 February 2021

Publisher's Note: MDPI stays neutral with regard to jurisdictional clai$\mathrm{ms}$ in published maps and institutional affiliations.

Copyright: (C) 2021 by the authors. Licensee MDPI, Basel, Switzerland. This article is an open access article distributed under the terms and conditions of the Creative Commons Attribution (CC BY) license (https:// creativecommons.org/licenses/by/ $4.0 /)$.
National Center of Rapeseed Improvement, National Key Laboratory of Crop Genetic Improvement, Huazhong Agricultural University, Wuhan 430070, China; xk@webmail.hzau.edu.cn (K.X.); wuyujin@webmail.hzau.edu.cn (Y.W.); sjr2015@webmail.hzau.edu.cn (J.S.); hukaining@gmail.com (K.H.); wuzengx@webmail.hzau.edu.cn (Z.W.); wenjing@mail.hzau.edu.cn (J.W.); yibin@mail.hzau.edu.cn (B.Y.); yuanbeauty@mail.hzau.edu.cn (C.M.); jxshen@mail.hzau.edu.cn (J.S.); futing@mail.hzau.edu.cn (T.F.)

* Correspondence: tujx@mail.hzau.edu.cn

\begin{abstract}
Photosystem II (PSII) is an important component of the chloroplast. The PSII repair cycle is crucial for the relief of photoinhibition and may be advantageous when improving stress resistance and photosynthetic efficiency. Lethal genes are widely used in the efficiency detection and method improvement of gene editing. In the present study, we identified the naturally occurring lethal mutant 7-521Y with etiolated cotyledons in Brassica napus, controlled by double-recessive genes (named cyd1 and cyd2). By combining whole-genome resequencing and map-based cloning, CYD1 was fine-mapped to a $29 \mathrm{~kb}$ genomic region using 15,167 etiolated individuals. Through cosegregation analysis and functional verification of the transgene, BnaC06.FtsH1 was determined to be the target gene; it encodes an filamentation temperature sensitive protein $\mathrm{H} 1$ (FtsH1) hydrolase that degrades damaged PSII D1 in Arabidopsis thaliana. The expression of BnaC06.FtsH1 was high in the cotyledons, leaves, and flowers of B. napus, and localized in the chloroplasts. In addition, the expression of EngA (upstream regulation gene of FtsH) increased and D1 decreased in 7-521Y. Double mutants of FtsH1 and FtsH5 were lethal in A. thaliana. Through phylogenetic analysis, the loss of FtsH5 was identified in Brassica, and the remaining FtsH1 was required for PSII repair cycle. CYD2 may be a homologous gene of FtsH1 on chromosome A07 of B. napus. Our study provides new insights into lethal mutants, the findings may help improve the efficiency of the PSII repair cycle and biomass accumulation in oilseed rape.
\end{abstract}

Keywords: lethal mutant; map-based cloning; FtsH1 hydrolase; PSII repair cycle

\section{Introduction}

The discovery and utilization of lethal genes is of great significance to the progress of molecular breeding. Lethal genes are widely used to detect the efficiency of gene editing systems in plant, which the editing efficiency can be observed easily at the seedling stage. As a powerful tool for genome editing, the CRISPR-Cas9 system was first successfully applied to plants by editing an albino gene OSPDS in rice, which exhibited an expected phenotype in $\mathrm{T}_{0}$ generation [1]. Using the $z b 7$ gene as an editing target, a large number of lethal seedlings observed in $\mathrm{T}_{0}$ generation, which proved that the dmc1 promotercontrolled (DPC) CRISPR/Cas9 is a high-efficiency editing system in maize [2]. Similar studies in Arabidopsis thaliana (A. thaliana) [3], Triticum aestivum [4], Gossypium spp. [5,6], Nicotiana tabacum [7], and other crops are often reported. However, few studies about gene cloning for lethality were reported for rapeseed due to the complex genome of Brassica napus (B. napus) and the limited reference genome before the release of B. napus, "ZS11". Therefore, the functional analysis of lethal mutants in rapeseed has potential value for improving gene editing efficiency and advancing technical innovation in molecular breeding. 
Approximately 1 to 1.5 billion years ago, chloroplasts evolved via the endosymbiosis of an ancient relative of an extant cyanobacterium, and they have become an indivisible part of the photosynthetic apparatus of terrestrial plants and algae [8,9]. The chloroplasts are largely responsible for photosynthesis, carbon metabolism, and fatty acid synthesis. However, the chloroplast genome is very small, and $95 \%$ of chloroplast proteins are encoded by nuclear genes [10]. The coordination of nuclear genes and chloroplast genes maintain the normal functions of chloroplasts, and the isolation and research of nuclear genes will contribute to further understanding the chloroplast developmental mechanism. Chloroplast-related mutants are excellent genetic resources to study the mechanisms of photosynthesis, chlorophyll biosynthesis, chloroplast structure, function, and development.

Photosystem II (PSII) is one of the most vulnerable parts of the plant photosynthesis system. Light energy is absorbed in the reaction center of the PSII complex, but it concurrently causes photodamage. A major target of this photodamage is the D1 protein of PSII, but the stability of PSII is necessary for the appropriate development of plants [11]. Thus, the PSII repair cycle is formed in photosynthetic organisms, and it involves the following processes: the partial decomposition of the PSII complex, selective degradation of photodamaged D1, synthesis of new D1, and reassembly of the functional PSII complex. The entire process is significant for the recovery of photosynthetic efficiency [12] In seed plants, Deg and filamentation temperature sensitive protein $\mathrm{H}(\mathrm{FtsH})$ proteases together complete the degradation of damaged D1 [13-16]. The A. thaliana FtsH gene family comprises 12 members (FtsH 1-12) and five FtsHi members which have lost the function for protein hydrolysis (FtsHi 1-5) [17]. However, FtsH members are the major thylakoid membrane proteases. Four FtsH proteins form a heterohexameric complex in the thylakoid membrane, comprising type A (includes FtsH5 and FtsH1) and type B (includes FtsH8 and $\mathrm{FtsH} 2$ ) [18]. At least one subunit of each type is required for chloroplast development and normal photosynthesis, and double mutants that result in the loss of either type A or type B proteases are lethal $[19,20]$. The appropriate turnover of FtsH is important for the maintenance of functional PSII repair. Overexpression of EngA (EngA-OX) results in the variegation of leaves and the accumulation of more cleaved D1 fragments. EngA may negatively regulate FtsH stability by interacting with its ATPase domain [21].

In this study, we identified a new lethal mutant 7-521Y with yellow cotyledon in B. napus. We fine-mapped and functional confirmed the candidate gene $C Y D 1$, which encodes an FtsH1 protease involved in the PSII repair cycle. Through phylogenetic analysis, we identified the loss of FtsH5 in Brassica; the remaining FtsH1 was required for plant survival. This study has important guiding significance for genotype selection of oilseed rape.

\section{Results}

\subsection{Characterization of the 7-521Y Mutant}

The natural mutant 7-521Y was found in the breeding material of the 7-521 line. The mutant had a yellow cotyledon, could not grow true leaves, and died after 7-14 d of growth (Figure 1a,b). The chlorophyll a, chlorophyll b, and carotenoid (Car) contents of the etiolated seedlings were significantly lower than those of normal 7-521G individuals (Figure 1c). No significant difference was detected in the chlorophyll a/b ratio between 7-521G $(3.920 \pm 0.054, \mathrm{mg} / \mathrm{mg})$ and 7-521Y $(4.468 \pm 0.465, \mathrm{mg} / \mathrm{mg})$, but the carotenoid/chlorophyll ratio of 7-521Y $(0.592 \pm 0.068, \mathrm{mg} / \mathrm{mg})$ was significantly higher than that of 7-521G $(0.186 \pm 0.000, \mathrm{mg} / \mathrm{mg})$ (Figure $\mathrm{S} 1)$. The chloroplast structures of 7-521G and 7-521Y were analyzed by transmission electron microscopy (TEM) (Figure 1d). The chloroplasts of 7-521G developed large starch grains; however, the chloroplasts of 7$521 Y$ did not develop any starch grain. Plump chloroplast structures and stacked thylakoid membranes were observed in 7-521G, but were not observed in 7-521Y (Figures 1d and S2). 
a

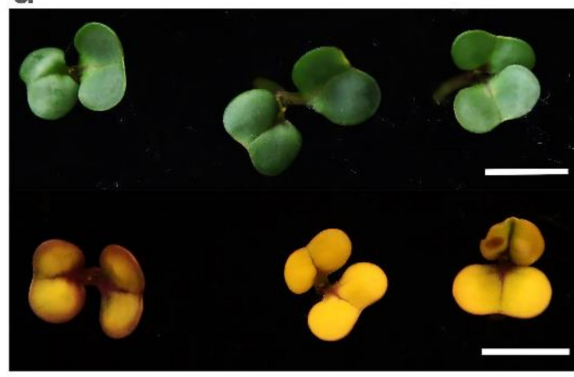

C

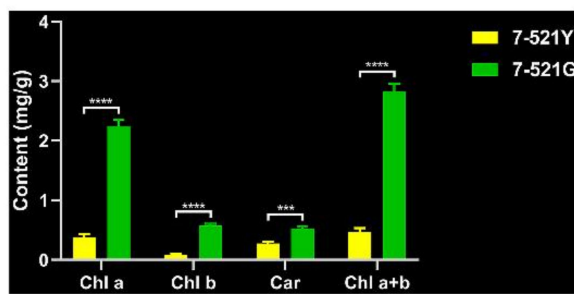

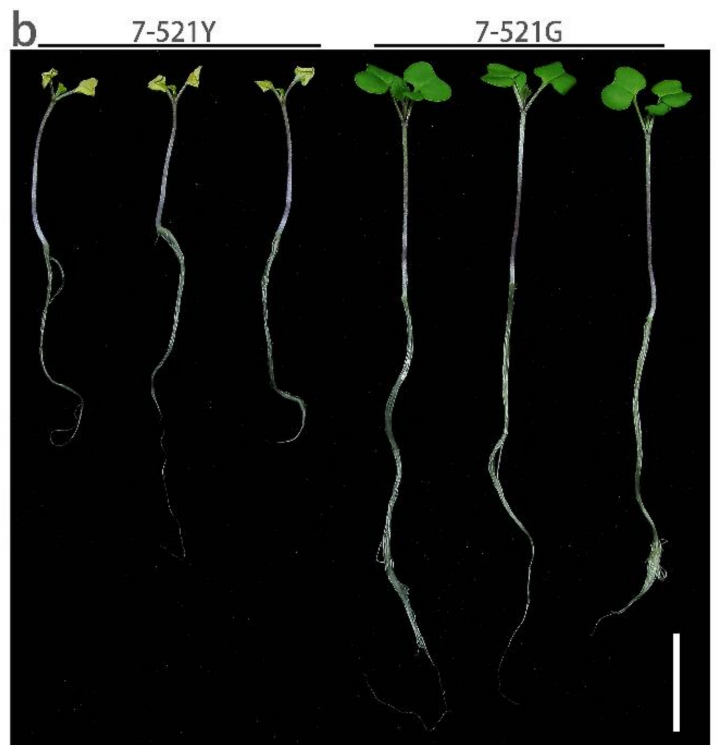

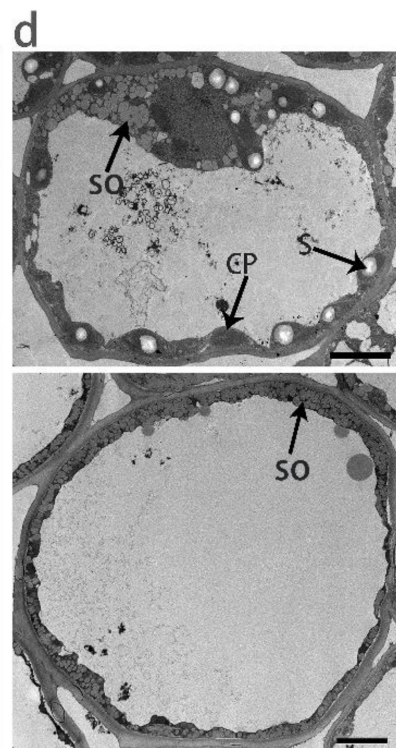

Figure 1. Phenotype characterization of 7-521Y. (a) Cotyledon phenotype of 7-521G (up) and 7-521Y (down) in the seedling stage (after 5 days of germination). (b) Whole plant phenotype of 7-521G (three on the right) and 7-521Y (three on the left) after germination for 12 days. (c) Chlorophyll $a$, chlorophyll b, carotenoid, and chlorophyll a+b content in 7-521Y and 7-521G. Car/Chl represents the carotenoid/chlorophyll ratio. Asterisks indicate a significant difference: ${ }^{* * *} p<0.001$ and **** $p<0.0001$ (Student's $t$-test). (d) Transmission electron microscope (TEM) micrographs of the cotyledons from 7-521G (up) and the mutant 7-521Y (down). Black arrows point to different structures in the cell. S-starch grains, CP-chloroplast, and SO—seed oil. Bars: (a) $5 \mathrm{~mm}$; (b) $2 \mathrm{~cm}$; (d) $5 \mu \mathrm{m}$.

Among the 484 progenies of 7-521, there were 123 etiolated seedlings and 361 green seedlings, with the genetic segregation ratio of $3: 1\left(\chi^{2}=0.025<\chi^{2}{ }_{0.05,1}=3.84\right)$. To verify that this trait of etiolated cotyledons was controlled by one or two genes, the heterozygous 7-521G was utilized as the paternal line in a cross with the Bing 409 line, and then the segregation of eight $F_{2}$ populations using seedling color was analyzed. Five out of eight $F_{2}$ populations showed a Mendelian segregation ratio of 15:1 (Table S1), which fits with a twogene model. The controlling genes were named CYD1 and CYD2. Double-recessive genes controlled this lethal trait, 7-521 carried heterozygous alleles for CYD1 and homozygous recessive alleles for $C Y D 2$.

\subsection{Whole-Genome Resequencing and Gene Mapping for the Lethal Gene CYD1}

Separately mixed normal and yellow plants in the 7-521 line were used for wholegenome resequencing, for which the average sequencing coverage depths were $52.67 \times$ and $56.22 \times$ in the 7-521G and 7-521Y pools, respectively. Resequencing generated approximately $93.5 \mathrm{~Gb}$ of clean data after filtering $102.3 \mathrm{~Gb}$ of raw data that consisted of 303,754,772 reads from 7-521G and 324,204,540 reads from 7-521Y. Based on the $\Delta$ SNP-index value, 4,177,432 SNPs were called, and a Manhattan map (Figure 2a) was used to illustrate each chromosome. Three potential regions were identified: chromosome A03 (6.85-6.90 Mb), chromosome A09 (22.78-22.90 Mb), and chromosome C06 (20.48-36.40 Mb). Indel markers were designed to screen 7-521G and 7-521Y DNA pools to identify polymorphic markers. Only markers on chromosome C06 showed polymorphisms between the two DNA pools. All results indicated that the major candidate gene $C Y D 1$ of 7-521 may be on chromosome C06 of B. napus (Figure 2b). 


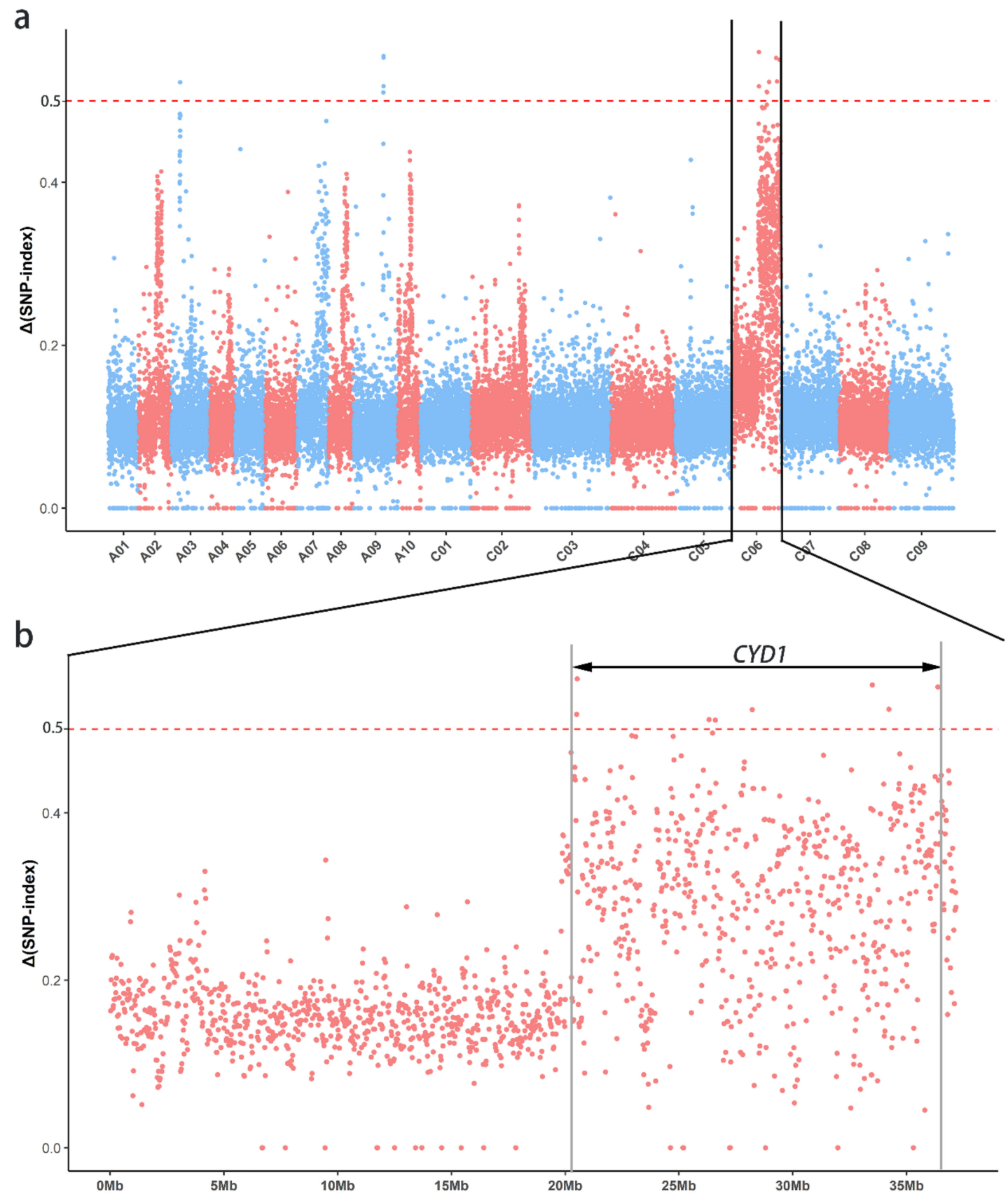

Figure 2. Candidate regions of CYD1 identified by whole-genome resequencing. (a) The x-axis represents the 19 chromosomes of Brassica napus arranged by physical position. The y-axis represents the value of $\Delta$ (SNP index), which was calculated by subtracting the SNP index of 7-521Y from that of 7-521G. (b) Enlarged view of chromosome C06. The candidate gene interval (marked by arrow) was determined using 0.5 as the threshold, and chromosome A03 and chromosome A09 were excluded through marker development.

To reveal the molecular mechanisms of the etiolated-cotyledon phenotype, we isolated the key gene CYD1 using map-based cloning, and SSR markers were designed. We selected 8777 individuals with etiolated cotyledons to map CYD1. CYD1 was initially located on chromosome C06, an interval between markers S361 and S342 (Figure 3a), and corresponding to a $428 \mathrm{~kb}$ region of the B. napus ZS11 physical map. Using a larger population of 15,167 etiolated seedlings, $C Y D 1$ was then fine-mapped to a $29 \mathrm{~kb}$ genomic region between SSR markers S481 and S342 (Figure 3b). The candidate interval contained five annotated open reading frames in the ZS11 reference genome (Figure 3c). Annotation of these 
genes (Table 1) indicated that BnaC06G0341000ZS was highly orthologous to AT1G50250 in A. thaliana. AT1G50250 encodes FtsH1 that regulates the PSII repair cycle, and FtsH1/FtsH5 double mutants had the etiolated-cotyledon phenotype.

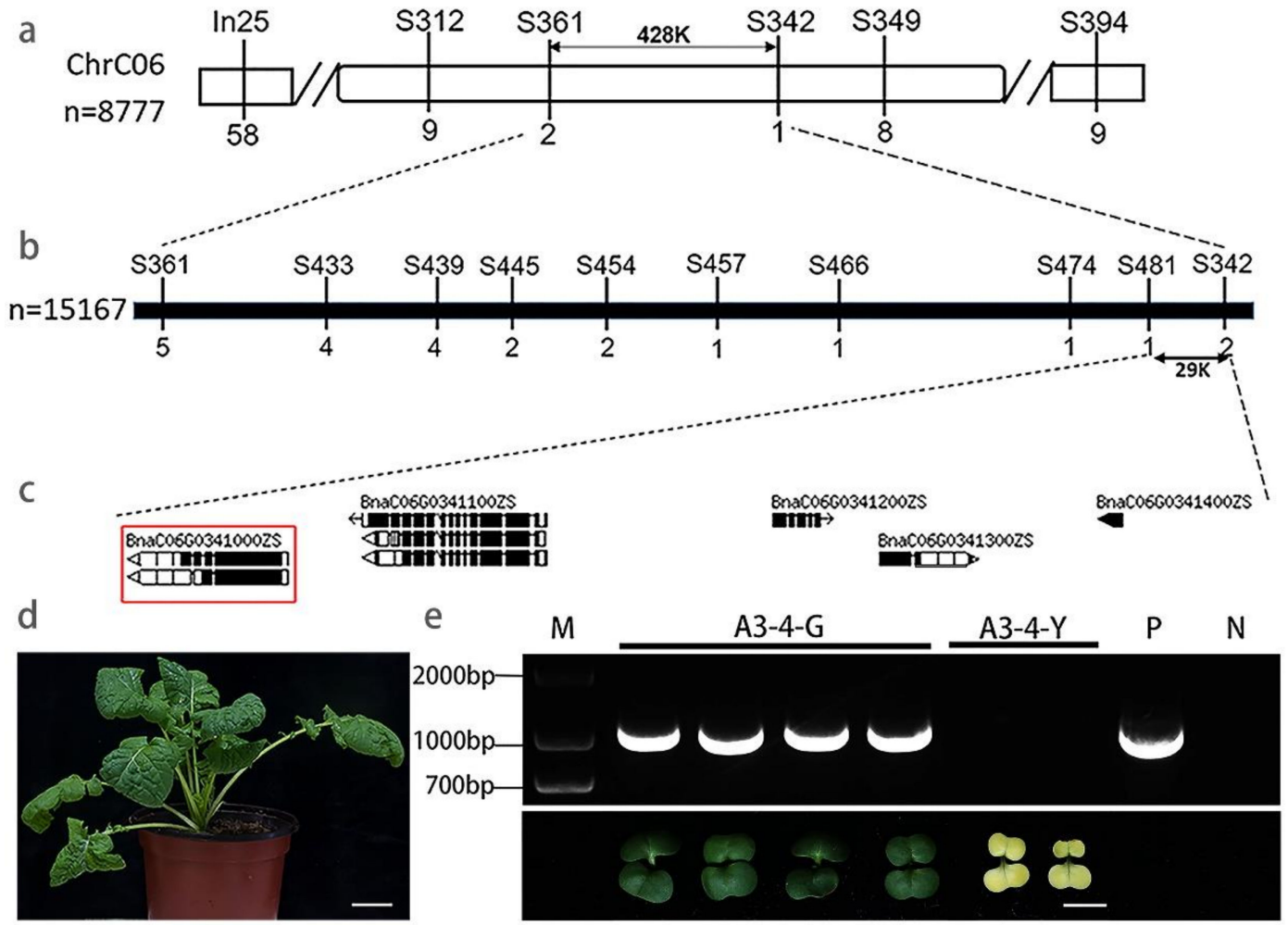

Figure 3. Fine mapping and functional confirmation of BnaC06.FtsH1 in rapeseed. (a) Initial mapping of CYD1 using 8777 etiolated-cotyledon individuals from the selfing segregation population of 7-521. The applied markers are marked above the chromosome, and the number of recombinants in this population is marked below. (b) Fine mapping of CYD1 using 15,167 etiolated individuals. The numbers indicate the number of recombinants. (c) Annotation in $29 \mathrm{~K}$ region according to the ZS11 (Brassica napus cultivar) reference genome. The red box indicates the candidate genes predicted in ZS11 database. (d) Confirmation of the BnaC06.FtsH1 function using the expression of BnaC06.FtsH1 in 7-521Y. The phenotype of the $\mathrm{T}_{0}$ transgenic plants returned to normal. (e) Identification of the $T_{1}$ transgenic seedlings using PCR. A3-4-G, normal individuals from the A3-4 T1 population; A3-4-Y, etiolated individuals from the A3-4 T1 population; N, negative control from 7-521Y; $\mathrm{P}$, positive control from vector plasmid; M, molecular markers. Bars: (d) $4 \mathrm{~cm}$; (e) $5 \mathrm{~mm}$.

Table 1. Prediction and annotation of candidate genes within the mapping region.

\begin{tabular}{cccc}
\hline Gene of B. napus & Chromosome Position & $\begin{array}{c}\text { Orthologous Gene of } \\
\text { A. thaliana }\end{array}$ & Annotation \\
\hline BnaC06G0341000ZS & $44,665,913-44,669,710$ & AT1G50250 & FtsH protease 1 \\
\hline BnaC06G0341100ZS & $44,671,450-44,675,781$ & AT1G67720 & $\begin{array}{c}\text { Leucine-rich repeat protein } \\
\text { kinase family protein }\end{array}$ \\
\hline BnaC06G0341200ZS & $44,681,149-44,682,236$ & AT4G01935 & insulin-induced protein \\
\hline BnaC06G0341300ZS & $44,683,655-44,685,971$ & AT1G67730 & beta-ketoacyl reductase 1 \\
\hline BnaC06G0341400ZS & $44,688,777-44,689,358$ & AT1G67740 & $\begin{array}{c}\text { PsbY precursor } \\
\text { (psbY) mRNA }\end{array}$ \\
\hline
\end{tabular}

A comparison of BnaC06.FtsH1 between 7-521G and 7-521Y revealed that BnaC06.FtsH1 was missing in etiolated individuals. To confirm if the deletion cosegregated with etiolated cotyledons, SCAR markers FtsH1-C6GFL-F and R, were developed on the full-length genomic DNA (gDNA) of BnaC06.FtsH1 (Figure S3). This marker completely cosegregated 
with the etiolated cotyledons of 15,167 individuals. Therefore, it can be speculated that BnaC06.FtsH1 is a candidate gene of CYD1.

\subsection{Functional Confirmation by B. napus Transformation}

To confirm whether the deletion of BnaC06.FtsH1 was the causal mutation, the allele BnaC06.FtsH1 of 7-521G driven by native promoter was cloned and introduced into the calli of the etiolated seedlings using $A$. tumefaciens-mediated transformation. Six positive seedlings were obtained by PCR identification; these positive seedlings were similar to 7-521G in their normal growth, development, and reproduction (Figure 3d). Phenotypic segregation occurred in the transgenic $\mathrm{T}_{1}$ generation, and the PCR results showed that all the recovered phenotypes were positive seedlings and etiolated individuals were negative seedlings (Figure 3e). These results suggest that the deletion of BnaC06.FtsH1 was responsible for the etiolated-cotyledons observed in 7-521Y.

\subsection{Subcellular Location and Expression of BnaC06.FtsH1}

The expression of BnaC06.FtsH1 was detected by qRT-PCR. According to the results, the transcription level of BnaC06.FtsH1 was low in the roots, hypocotyls, stems, flower buds, and siliques, but high in the cotyledons, leaves, and flowers (Figure 4a). Driven by the BnaC06.FtsH1 native promoter, the GUS reporter gene was expressed in A. thaliana to monitor the expression of BnaC06.FtsH1. Multiple transgenic plants were obtained, and the GUS staining results showed that BnaC06.FtsH1 was expressed in almost all tissues including cotyledons, young leaves, and flowers, and this was almost consistent with the qRT-PCR results (Figure $4 b-f)$.

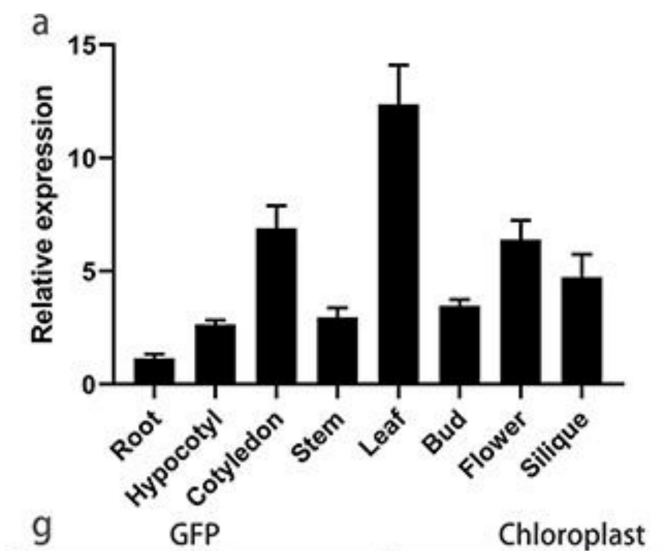

b c

c d
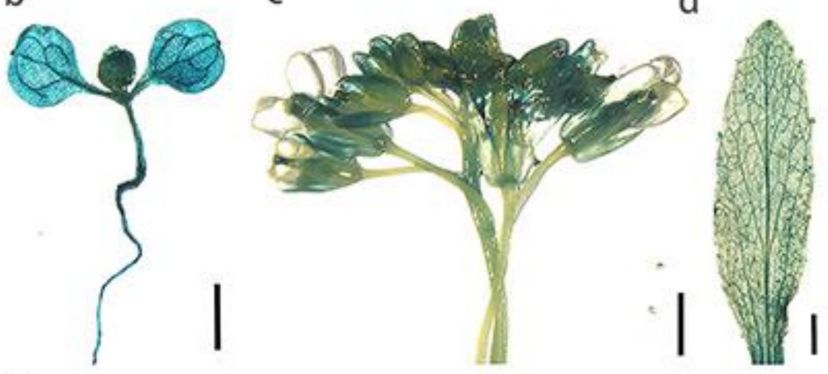

e

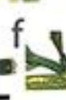

Bright field
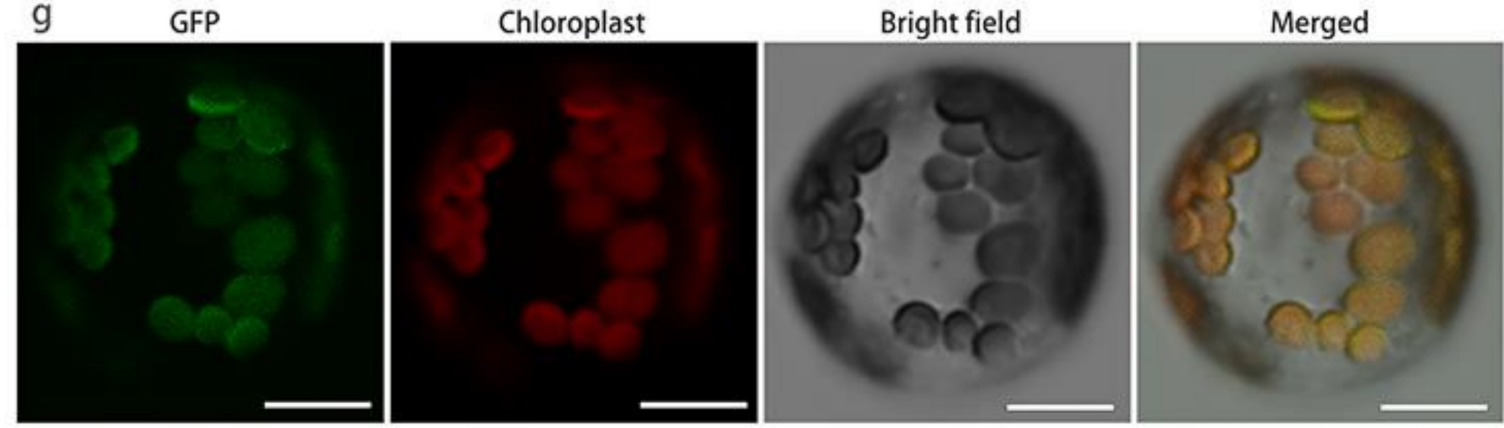

Figure 4. Subcellular localization and expression pattern of BnaC06.FtsH1. (a) Expression pattern of BnaC06.FtsH1 detected by qRT-PCR in the hypocotyls, roots, stems, cotyledons, leaves, flower buds, siliques, and flowers of the Brassica napus cultivar Westar, using the expression of BnaENTH as a reference. Values are expressed as average \pm SD $(n=3)$. (b-f) GUS staining analysis of BnaC06.FtsH1. (b) Tender seedling, (c) bud, (d) leaf, (e) silique, and (f) stem. (g) Subcellular localization of BnaC06.FtsH1 in A. thaliana protoplasts. From left to right: green fluorescence of BnaC06.FtsH1; chloroplast spontaneous red fluorescence; bright-field image; merged image of the first three images. Bars: (b-f) $1 \mathrm{~mm}$; (g) $12 \mu \mathrm{m}$. 
To explore the subcellular localization of BnaC06.FtsH1, GFP was fused to the Cterminus driven by the $35 \mathrm{~S}$ promoter and transiently expressed in Arabidopsis mesophyll protoplasts. As expected, the BnaC06.FtsH1 signal closely overlapped with the chlorophyll autofluorescence signal, illustrating that BnaC06.FtsH1 was localized in the chloroplasts (Figure 4g).

\subsection{Deletion of CYD1 Influences the Expression of EngA and PsbA in B. napus}

Through sequence alignment, we identified four homologs of EngA (AT3G12080) in B. napus ZS11: two in the A genome (BnaA05G0428200ZS and BnaA01G0369900ZS) and two in the C genome (BnaC05G0481000ZS and BnaC01G0461600ZS). The structural diagram for the four genes was mapped (Figure 5a). The gene sequences of BnaA01G0369900ZS and BnaC01G0461600ZS were too short, and their mRNA expression could be hardly detected in 7-521G or 7-521Y. However, the expression of homologs in A05 and C05 was higher in the 7-521Y individuals than in the 7-521G individuals (Figure 5b). There are two homologs of PsbA (the coding gene of D1) in ZS11: BnaA01G0425500ZS and BnaA06G0201300ZS. The CDS sequences of the two copies were identical, and we could not design-specific primers to distinguish them. However, the total expression of Bna.PsbA was relatively decreased in the 7-521Y individuals (Figure 5b). This indicates that the deletion of CYD1 increased the expression of $E n g A$ and decreased the expression of $P s b A$, and that the three genes may interact in B. napus.

a

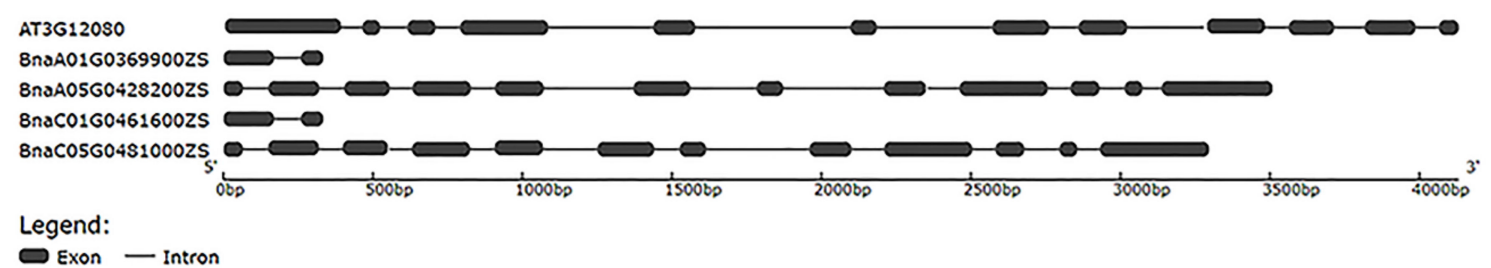

b

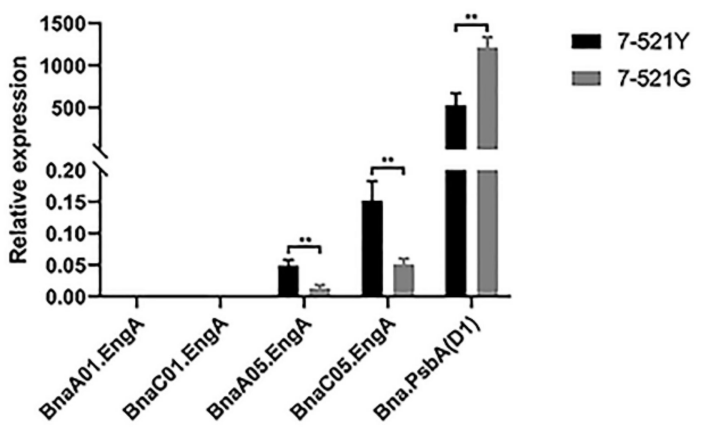

Figure 5. Expression levels of EngA and PsbA determined by qRT-PCR in Brassica napus. (a) Homologous gene structure analysis of EngA in B. napus. (b) Relative expression of EngA and PsbA homologous genes in B. napus. ${ }^{* *}$ Denotes significant differences, $p<0.001$, Student's $t$-test.

\subsection{CYD2 May Be the Homologous Gene of FtsH1 on Chromosome A07}

A previous study has indicated that double mutants with complete losses of either type B ( $\mathrm{FtsH} 8$ and $\mathrm{FtsH} 2$ ) or type A (FtsH5 and FtsH1) were lethal [20]. The full-length protein sequences of these four genes and their eigth closest homologs were extracted for further phylogenetic analysis (Figure 6a). These results showed that $\mathrm{FtsH} 2$ and $\mathrm{FtsH} 8 \mathrm{had} 2$ and 4 homologous genes in ZS11, respectively. There were six FtsH homologous copies of the type B proteins to help maintain the stability of the PSII repair cycle in B. napus. However, there was no homologous gene of FtsH5 in ZS11, and only two copies of FtsH1 on chromosome $\mathrm{C} 06$ and chromosome A07 determine the normal function of type A proteins. We speculated that the 7-521Y mutant was caused by the abnormal function of type A 
proteins. SSR markers were designed on the homologous segment of chromosome A07 and multiple polymorphic markers were found (Figure S4). We preliminarily concluded that CYD2 is located on chromosome A07 and may be the homologous gene of FtsH1.

a

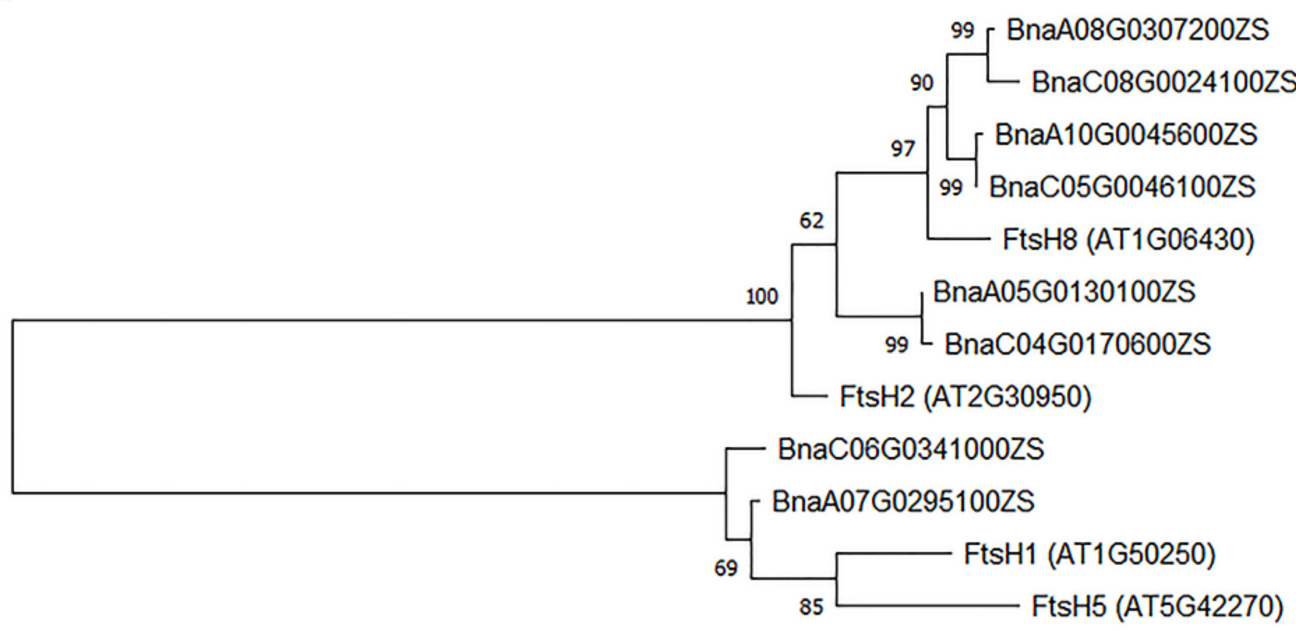

0.10

b

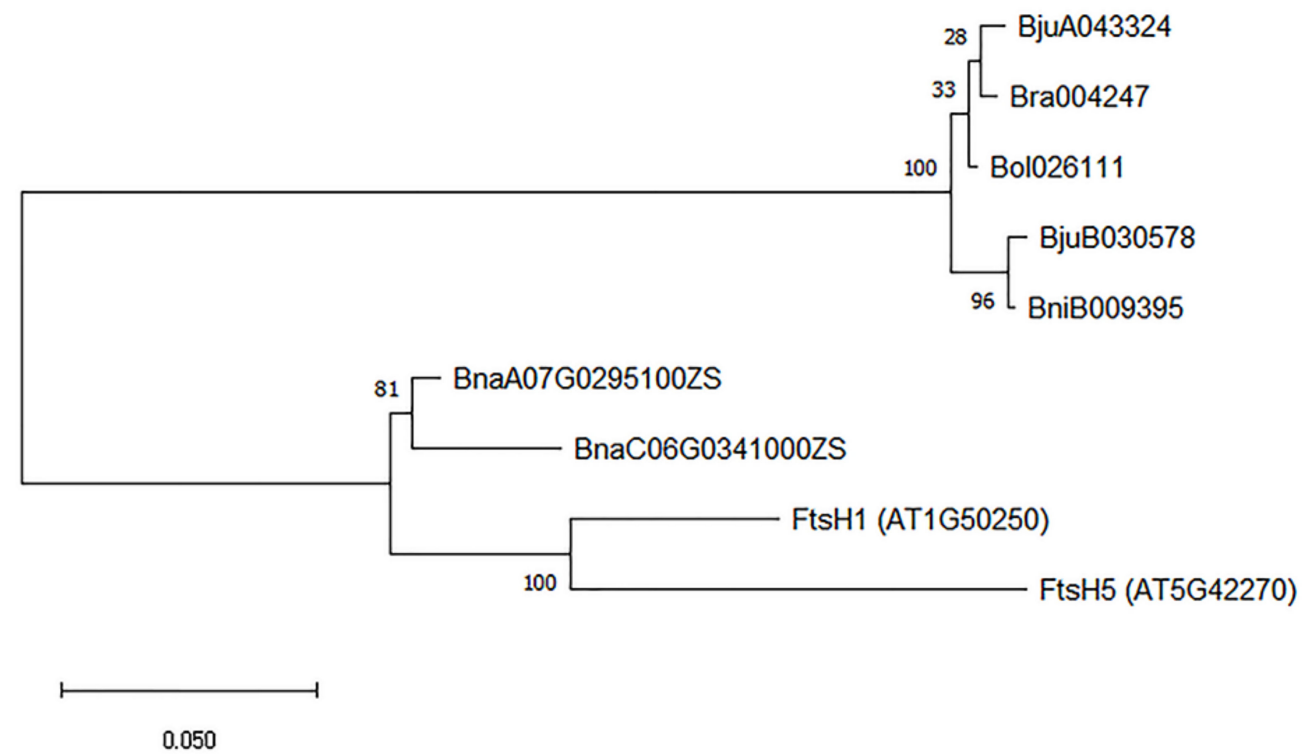

Figure 6. Construction of two maximum likelihood phylogenetic trees. (a) Phylogenetic analysis of filamentation temperature sensitive protein $\mathrm{H} 5$ (FtsH5), FtsH1, FtsH8, FtsH2, and their 8 closest homologs in Brassica napus. (b) Phylogenetic analysis of type A (FtsH1 and FtsH5) and their closest homologs in B. rape, B. nigra, B. juncea, B. oleracea, and B. napus. The tree is drawn to scale, with branch lengths measured as the number of substitutions per site. The length of these branches refers to the rate of sequence variation. Numbers represent the reliability calculated by the bootstrap test with 1000 replicates.

\subsection{Phylogenetic Analysis of FtsH1 and FtsH5 in Brassica}

To further illustrate the impacts of the loss of $\mathrm{FtsH} 5$, the full-length protein sequences of type A (FtsH1 and FtsH5) and their closest homologs in Brassica rape (B. rape), Brassica nigra (B. nigra), Brassica oleracea (B. oleracea), Brassica juncea (B. juncea), and B. napus were used for phylogenetic analysis (Figure $6 \mathrm{~b}$ ). The results indicated that there was only one homologous 
gene of FtsH1 and FtsH5 in Brassica diploids (B. rape, B. nigra, and B. oleracea), and two homologs in Brassica allotetraploids (B. juncea and B. napus). These seven genes all had greater sequence similarity to FtsH1. Brassica diploids had only one FtsH1 homologous gene of type A for the formation of a heterohexameric complex and a PSII repair cycle.

\section{Discussion}

In this study, we characterized a natural mutant with etiolated cotyledons named 7-521Y, which grew abnormally and could not survive long term. Instead of conventional strategies for gene location using backcross $(\mathrm{BC})$ and $\mathrm{F}_{2}$ populations, we directly used heterozygous selfing lines of 7-521 to locate CYD1. The mapping population often determines the frequency of exchange at a certain site [22]. Restricted by mapping population possibly, we used a large group of 15,167 lethal seedlings to fine-map CYD1 to a $29 \mathrm{~kb}$ interval. Because 7-521y could not grow normally, it was impossible to obtain homozygous lethal mutants; therefore, we adjusted the traditional tissue culture method [23]. We planted a large number of heterozygous lines in $\mathrm{M}_{0}$, which were cultured in dark conditions for $5 \mathrm{~d}$ for the elongation of hypocotyls, and then transferred to light condition for $1 \mathrm{~d}$ to distinguish the mutant phenotype from the normal phenotype. Etiolated seedlings were cut for complementary experiments, and BnaC06.FtsH1 was determined as the candidate gene. The results of this study will be significant for future research on lethal mutants.

FtsH plays a crucial role in the maintenance of thylakoid membranes [24]. FtsH1 participates in the formation of the hetero-hexameric complex and regulation of thylakoid membrane biogenesis [25]. Meanwhile, thylakoid membranes are densely packed with pigment-protein complexes of PSII and PSI [26], lack of chlorophyll and carotenoids in the mutant may cause PSII and chloroplast abnormalities. Therefore, plump chloroplast structures and stacked thylakoid membranes were not observed in mutant 7-521Y. The ratio of Car/Chl is frequently applied to assess stress tolerance of photosynthesizing tissues [27], shift of Car/Chl ratio in mutant may be an indicator of functional PSII dissasembly.

In photosynthetic organisms, the thylakoid $\mathrm{FtsH}$ complexes participate in the process of PSII repair cycle, particularly the degradation of damaged D1 [12,28,29]. CYD1 may encode an FtsH1 hydrolase that is responsible for the degradation of D1. In the 7-521 strain, the deletion of CYD1 in the mutant prevents the degradation of the damaged D1, and new D1 structural proteins cannot be synthesized, which blocks the PSII repair cycle for photosynthesis and causes the death of the mutant. However, the FtsH1 hydrolase only removes the light-damaged D1 protein and does not bind to the normal D1 protein [30]. We could not verify the interactions of BnaC06.FtsH1 and D1 using the yeast two-hybrid assays. However, we found that the loss of CYD1 increased the expression of BnaA05.EngA and BnaC05.EngA and decreased the expression of D1. EngA may negatively regulate the stability of FtsH1 and affect the degradation of damaged D1 by FtsH1 in B. napus [21].

Six Brassica species constitute the recognized "triangle of $U$ " model [31], including three diploid species-B. rapa (A genome), B. nigra (B genome), and B. oleracea (C genome)and three amphidiploid species resulting from pairwise hybridization-B. napus (AC genome), B. juncea (AB genome), and Brassica carinata (BC genome). B. rapa and B. oleracea originated from a common ancestor, and their genomes are very similar [32]. Therefore, the recessive traits controlled by two genes are often controlled by two duplicate genes in B. napus [31]. We speculated that CYD2 may be a duplicated gene of CYD1 on chromosome A07. Through marker development, it was confirmed that CYD2 was located on chromosome A07 where BnaA07.FtsH1 is located. CYD2 may be BnaA07.FtsH1, but further verification is required. According to our results, there was a homologous gene of FtsH1 in B. rapa and B. oleracea, respectively. BnaC06.FtsH1 may have evolved from Bol026111 (the orthologs of FtsH1 in B. oleracea), while BnaA07.FtsH1 may have evolved from Bra004247 (the orthologs of FtsH1 in B. rapa).

In A. thaliana, FtsH1 and Fts $\mathrm{H} 5$ together determined the normal function of type A FtsH hydrolase, and we found that it was essential for at least one subunit of the PSII repair cycle [20]. Through phylogenetic analysis, we identified the loss of FtsH5 in Brassica. 
In B. juncea and B. napus, the composition of type A proteins changed into two FtsH1 homologous genes to ensure the normal degradation of the damaged D1. However, in B. rapa, B. nigra, and B. oleracea, type A consisted of only one FtsH1 homologous gene, which was extremely detrimental to the survival of the plants. Any form of mutation in the FtsH1 homologous genes may cause a lethal mutation phenotype similar to 7-521Y in Brassica diploid. This indicates that this gene is essential for the PSII repair cycle and the survival and normal growth of Brassica.

Although details of PSII repair cycle are still controversial, preventing photoinhibition is considered as a means to improve stress resistance and photosynthetic efficiency. Coordinating the degradation of D1 is considered an effective way to alleviate photoinhibition [33]. According to a recent study, the overexpression of SIWHY1 enhances the synthesis of D1 in PSII and increases the chilling resistance of tomato [34], and increasing the expression of $\mathrm{D} 1$ via the nucleus can significantly increase the photosynthetic efficiency of $A$. thaliana, tobacco and yield of rice [35]. The deletion of CYD1 decreased the expression of D1 in this study. It may be possible to adjust the expression of $C Y D 1$ to accelerate the degradation of damaged D1 to improve the efficiency of the PSII repair cycle, thereby promoting photosynthesis and biomass accumulation in B. napus. In this study, we cloned BnaC06.FtsH1 and identified the loss of FtsH5 in B. napus. We then speculated that the lethal phenotype of 7-521Y was caused by BnaC06.FtsH1 and BnaA07.FtsH1. Because of the adjustment in our experimental methods, our research has reference significance for research on other lethal mutants. The mechanism analysis of 7-521Y also has potential value for the innovation of molecular breeding technology, especially gene editing in B. napus.

\section{Materials and Methods}

\subsection{Genetic Analysis and Related Plant Materials}

The mutant 7-521Y was found in the breeding material line 7-521 and mutated naturally. Green plants with heterozygous genotypes were selfed to obtain families, which were then used as segregating populations for mapping CYD1 (Figure S5). The heterozygous genotype plants were then crossed with Bing409 to obtain $F_{1}$ plants, which were self-crossed to obtain $F_{2}$ segregation for analyzing heredity patterns. Plant descendants segregated at the chromosome A07 locus but with a recessive homozygous allele on the chromosome $\mathrm{C} 06$ locus were used to obtain $\mathrm{F}_{3}-1$ populations, which were used for fine mapping CYD2. All B. napus materials were planted in the experimental base at Huazhong Agricultural University in Wuhan. The planting density between rows was $20 \mathrm{~cm}$ and the row spacing was $30 \mathrm{~cm}$.

\subsection{Pigment Assay}

Fresh cotyledons (approximately $0.15 \mathrm{~g}$ ) were cut into pieces and placed into $10 \mathrm{~mL}$ of the extraction solution $(96 \%$ ethanol: acetone $=1: 8(v / v))$, and then placed in the dark for $48 \mathrm{~h}$. The pigment content was determined spectrophotometrically (Mapada, Shanghai, China), quartz cuvettes with $10 \mathrm{~mm}$ optical pathlength were used. The absorbance of chlorophyll solution was determined at 470,645 , and $663 \mathrm{~nm}$, with the extraction solution as the control [36]. Three replicates were assessed, and the results were calculated using the following equations [30]:

$$
\begin{gathered}
\text { Chl a }=\left(12.21 \mathrm{~A}_{663}-2.81 \mathrm{~A}_{645}\right) /(50 \times \text { weight }), \mathrm{Chl} \mathrm{b}=\left(20.13 \mathrm{~A}_{645}-5.03 \mathrm{~A}_{663}\right) /(50 \times \text { weight }) \\
\text { Car }=\left(1000 \mathrm{~A}_{470}-3.27\left(12.21 \mathrm{~A}_{663}-2.81 \mathrm{~A}_{645}\right)-104\left(20.13 \mathrm{~A}_{645}-5.03 \mathrm{~A}_{663}\right)\right) /(11450 \times \text { weight })
\end{gathered}
$$

where $\mathrm{Chl} \mathrm{a}, \mathrm{Chl} b$, and Car represent the contents of chlorophyll a, chlorophyll b, and Carotenoid in leaves (mg/g), respectively. while $\mathrm{A}_{663}, \mathrm{~A}_{645}$, and $\mathrm{A}_{470}$ represent the absorbance at 663,645 , and $470 \mathrm{~nm}$, respectively. Weight is the weight of cotyledons (g).

\subsection{Transmission Electron Microscope (TEM) Analysis}

Fresh cotyledons of 7-521G and mutant plants 7-521Y were cut and fixed in a solution of $0.1 \mathrm{M}$ phosphate buffer of $\mathrm{pH}$ of $7.4 \%$ and $2.5 \%$ glutaraldehyde $(w / v)$; TEM analysis 
was then performed by H-7650 (Hitachi, Tokyo, Japan). The experiment was performed as described previously [37].

\subsection{Whole-Genome Resequencing and Gene Mapping}

Genomic DNA from 7-521G and 7-521Y bulks was subjected to whole-genome resequencing. The total DNA was extracted from cotyledons using the Plant Genomic DNA Kit (Tiangen, Beijing, China). To ensure the concentration and quality of gDNA, assessments were performed by Invitrogen Qubit 2.0 (Thermo Fisher Scientific, Waltham, MA, USA). Two DNA pools were used for the construction of libraries using TruSeq DNA Sample Preparation Kits (Illumina, San Diego, CA, USA). Then, a HiSeq 2000 system (Illumina, San Diego, CA, USA) was used to produce paired-end reads. Quality analysis and filtering of read datasets were performed using FastQC (http:/ / www.bioinformatics.babraham.ac.uk/ projects / fastqc/, last accessed date 7 December 2020) and Trimmomatic software [38], respectively. While IGV [39] was used as the genome browser. SNP and INDEL variant calling was performed using a previously described method [40], with the reference genome of $B$. napus "Darmor-bzh" [32]. The $\Delta$ (SNP index) was calculated by subtracting the SNP index of 7-521Y from that of 7-521G, and a Manhattan map was drawn using the ggpplot2 package in R [41]. The BSA method was applied to screen molecular markers, and the SSR markers were designed using an online tool (https:/ / bioinfo.inf.ufg.br/websat/, last accessed date 7 December 2020) and synthesized (Tsingke, Wuhan, China), utilizing the reference genome sequence of B. napus "ZS11" [42]. We used 15,167 etiolated plants to map CYD1, in which the genomic DNA was extracted using the CTAB (hexadecyltrimethylammonium bromide) protocol [43]. The candidate interval sequences were submitted to the BnPIR website [42] and the Brassica Database (BRAD) [44] for BLAST analysis. The putative functions of candidate genes were adopted from their orthologs in A. thaliana, using the BLAST tool of the TAIR website (https:/ / www.arabidopsis.org/, last accessed date 7 December 2020).

\subsection{Plasmid Construction and Transformation for Complementation}

To validate the function of BnaC6.FtsH1, a 6352-bp DNA fragment of the gene including the $2784 \mathrm{bp}$ upstream sequence, $2357 \mathrm{bp}$ coding region, and $1211 \mathrm{bp}$ downstream sequence of BnaC6.FTSH1 was amplified from 7-521G (primers FtsH1-CZQC-3F and FtsH1CZQC-3R) and then cloned into the PCAMBIA2300 vector [45] using single restriction endonucleases (Pst I) and a ClonExpress II One Step Cloning Kit (Vazyme, Nanjing, China). We confirmed that the constructed vectors were correct by restriction digestion analysis and sequencing. Finally, the vector plasmid was transferred into GV3101 (Agrobacterium tumefaciens strain) by the electroporation method, using Electroporator 2510 (Eppendorf, Hamburg, Germany) in $100 \Omega, 50 \mu \mathrm{F}$ and $1800 \mathrm{~V}$, and subsequently transferred into yellow individuals of the segregation lines. The hypocotyl of the mutants could be elongated in the dark. Tissue culture was performed as described previously [23]. We seeded the segregation population on $\mathrm{M}_{0}$ solid medium and cultured in the dark for 5-6 d, and then cultured under light condition for $1 \mathrm{~d}$. The yellow plants were selected for complementary transformation. Kalamycin was found to inhibit callus differentiation and plant regeneration in our materials; therefore, it was not used for selection during the tissue culture process. Positive seedlings were confirmed by PCR (marker M13-47F and A3-YXJC-11R), and the genetic background of the positive seedlings was found to be homozygous recessive using markers S445, S474, S342, and S349.

\subsection{RNA Extraction and Quantitative Real Time PCR ( $q R T-P C R$ )}

Different tissues of Westar were sampled for total RNA extraction, using the RNAprep Pure Plant Kit (Tiangen, Beijing, China), including the hypocotyls, roots, stems, cotyledons, leaves, buds, siliques, flowers of Westar, and cotyledons of 7-521G and 7-521Y. Three biological replicates were used for all samples. Reverse transcription was conducted using the RevertAid First Strand cDNA Synthesis Kit (Thermo Fisher, Massachusetts, USA). Real-time PCR was performed in triplicate using the SYBR Green Realtime PCR Master 
Mix (Toyobo, Tokyo, Japan) in a CFX96 Touch Real-Time PCR (Bio-Rad, California, USA). Quantitative RT-PCR measurements were obtained using the relative quantification $2^{-\Delta \Delta \mathrm{Ct}}$ method. Data are expressed as the mean of three biological replicates $\pm \mathrm{SD}$. All genespecific primers used for the amplification are listed in Table S2, and BnaENTH was used as an internal reference gene [46].

\subsection{Identification of Eng A and PsbA Homologs in B. napus}

Homologous genes of $E n g A$ and $P s b A$ in $B$. napus were identified, using orthologous and BLAST tools of the BnPIR website [42]. The structural diagram for the EngA homologs was mapped using GSDS 2.0 [47].

\subsection{Protein Subcellular Localization and GUS Staining}

The BnaC6.FtsH1-coding sequence (using primers C6SL-2F and C6SL-2R) was inserted into the CaMV $35 \mathrm{~S}$ promoter containing a pM999-GFP vector [45] using single restriction endonucleases (Xba I) and a ClonExpress II One Step Cloning Kit (Vazyme, Nanjing, China). The constructed vector was confirmed correct by sequencing and then introduced into A. thaliana (ecotype Col-0) protoplast by PEG-calcium-mediated transfection, then the transfected protoplasts were cultured for 5-24 h [48]. Chloroplast autofluorescence was determined, and the GFP signal was detected using a laser scanning confocal microscope FV1200 (Olympus, Tokyo, Japan) [49] with a green channel, $488 \mathrm{~nm}$ excitation wavelength, and a 500-530 nm emission wavelength, respectively.

The promoter containing a 661-bp upstream region of BnaC6.FtsH1 was amplified (primers C6-P661+Sal1-F and C6-Pro+BamH1-R) and cloned into the pCAMBIA2300 fused with GUS; the resulting plasmid was introduced into A. thaliana (ecotype Col-0). A. thaliana was transformed using the floral-dip method [50]. Different tissues of Arabidopsis $\mathrm{T}_{1}$ transgenic lines were stained overnight with X-Gluc solution at $37^{\circ} \mathrm{C}$, and then washed with ethanol as previously described [51].

\subsection{Phylogenetic Analysis}

Homologs of FtsH1, FtsH2, FtsH5, and FtsH8 were searched using the TAIR website (https:/ / www.arabidopsis.org/index.jsp, last accessed date 7 December 2020), the Brassica Database [44], and the BnPIR website [42]. Phylogenetic trees were constructed using MEGA X [52]. In detail, protein sequences were aligned using MUSCLE [53], and their evolutionary history was inferred using the maximum likelihood method [54] and a JonesTaylor-Thornton matrix-based model [55]. In the software interface, nearest-neighbor interchange was used for a heuristic search, while make initial tree automatically (Default$\mathrm{NJ} / \mathrm{BioNJ}$ ) was used to obtain the initial tree. To assess the reliability of the phylogenetic tree, a bootstrap test $[56,57]$ was performed with 1000 replicates.

Supplementary Materials: The following are available online at https:/ /www.mdpi.com/1422-006 7/22/4/2087/s1, Figure S1: Chlorophyll a/b ratio and carotenoid/chlorophyll ratio in 7-521Y and 7521G. The significant differences were identified using a Student's $t$-test: n.s., not significant; asterisks indicate a significant difference: $* * * p<0.001$. Figure S2. Transmission electron microscope (TEM) micrographs of the cotyledons from 7-521G and the mutant 7-521Y. Stacked thylakoid membranes were observed in 7-521G (left), but were not observed in 7-521Y (right). S: starch grains, TM: thylakoid membrane. Bars: $300 \mathrm{~nm}$. Figure S3. Genotype of the three recombinants between S481 and S342 in the 15167 etiolated individuals. Detected using the Scar marker on a $2 \%$ agarose gel. The final three recombinants were 2-31, 1-31, 2-82, using 7-521Y as negative control and 7-521G as positive control. Figure S4. Polymorphic markers on chrA07, ZG1-12 and ZY1-12 in $\mathrm{F}_{3}-1$ population were used as templates. Multiple polymorphic markers were found in the $\mathrm{F}_{3}-1$ segregating population. Normal phenotypes: ZG1-12, lethal phenotypes: ZY1-12. Figure S5. The flow diagram of population development. Table S1. Genetic analyses of the trait in $F_{2}$ Progenies. Table S2. Primers used in this study.

Author Contributions: K.X. and J.T. conceived and designed the study. J.W., B.Y., C.M., J.S. (Jinxiong Shen), and T.F. provided advice for the experimental design. K.X. performed most of the experiments 
and analyses and wrote the manuscript. Y.W. and J.S. (Jurong Song) participated in vector construction and genetic transformation. K.H. and Z.W. were involved in data processing. All authors reviewed and edited this manuscript. All authors have read and agreed to the published version of the manuscript.

Funding: This work was funded by the National Key Research and Development Program of China (Grant No. 2016YFD0100305).

Data Availability Statement: All data generated or analyzed during this study are included in this published article and its supplementary information files.

Conflicts of Interest: The authors declare that they have no conflict of interest.

\section{References}

1. Shan, Q.; Wang, Y.; Li, J.; Zhang, Y.; Chen, K.; Liang, Z.; Zhang, K.; Liu, J.; Xi, J.J.; Qiu, J.-L. Targeted genome modification of crop plants using a CRISPR-Cas system. Nat. Biotechnol. 2013, 31, 686-688. [CrossRef] [PubMed]

2. Feng, C.; Su, H.; Bai, H.; Wang, R.; Liu, Y.; Guo, X.; Liu, C.; Zhang, J.; Yuan, J.; Birchler, J.A. High-efficiency genome editing using a dmc1 promoter-controlled CRISPR/Cas9 system in maize. Plant Biotechnol. J. 2018, 16, 1848-1857. [CrossRef]

3. Ma, X.; Zhang, Q.; Zhu, Q.; Liu, W.; Chen, Y.; Qiu, R.; Wang, B.; Yang, Z.; Li, H.; Lin, Y. A robust CRISPR/Cas9 system for convenient, high-efficiency multiplex genome editing in monocot and dicot plants. Mol. Plant 2015, 8, 1274-1284. [CrossRef] [PubMed]

4. Li, J.; Zhang, S.; Zhang, R.; Gao, J.; Qi, Y.; Song, G.; Li, W.; Li, Y.; Li, G. Efficient multiplex genome editing by CRISPR/Cas9 in common wheat. Plant Biotechnol. J. 2020. [CrossRef]

5. Wang, P.; Zhang, J.; Sun, L.; Ma, Y.; Xu, J.; Liang, S.; Deng, J.; Tan, J.; Zhang, Q.; Tu, L. High efficient multisites genome editing in allotetraploid cotton (Gossypium hirsutum) using CRISPR/Cas9 system. Plant Biotechnol. J. 2018, 16, 137-150. [CrossRef] [PubMed]

6. $\quad$ Li, B.; Rui, H.; Li, Y.; Wang, Q.; Alariqi, M.; Qin, L.; Sun, L.; Ding, X.; Wang, F.; Zou, J. Robust CRISPR/Cpf1 (Cas12a)-mediated genome editing in allotetraploid cotton (Gossypium hirsutum). Plant Biotechnol. J. 2019, 17, 1862-1864. [CrossRef]

7. Ma, X.; Zhang, X.; Liu, H.; Li, Z. Highly efficient DNA-free plant genome editing using virally delivered CRISPR-Cas9. Nat. Plants 2020, 6, 773-779. [CrossRef]

8. Douzery, E.J.P.; Snell, E.A.; Bapteste, E.; Delsuc, F.; Philippe, H. The timing of eukaryotic evolution: Does a relaxed molecular clock reconcile proteins and fossils? Proc. Natl. Acad. Sci. USA 2004, 101, 15386-15391. [CrossRef] [PubMed]

9. Jarvis, P.; López-Juez, E. Biogenesis and homeostasis of chloroplasts and other plastids. Nat. Rev. Mol. Cell Biol. 2013, 14, 787-802. [CrossRef]

10. Koussevitzky, S.; Nott, A.; Mockler, T.C.; Hong, F.; Sachetto-Martins, G.; Surpin, M.; Lim, J.; Mittler, R.; Chory, J. Signals from chloroplasts converge to regulate nuclear gene expression. Science 2007, 316, 715-719. [CrossRef]

11. Murata, N.; Takahashi, S.; Nishiyama, Y.; Allakhverdiev, S.I. Photoinhibition of photosystem II under environmental stress. Biochim. Biophys. Acta 2007, 1767, 414-421. [CrossRef] [PubMed]

12. Jarvi, S.; Suorsa, M.; Aro, E.M. Photosystem II repair in plant chloroplasts-Regulation, assisting proteins and shared components with photosystem II biogenesis. Biochim. Biophys. Acta 2015, 1847, 900-909. [CrossRef]

13. Kato, Y.; Sun, X.; Zhang, L.; Sakamoto, W. Cooperative D1 degradation in the photosystem II repair mediated by chloroplastic proteases in Arabidopsis. Plant Physiol. 2012, 159, 1428-1439. [CrossRef]

14. Sun, X.; Fu, T.; Chen, N.; Guo, J.; Ma, J.; Zou, M.; Lu, C.; Zhang, L. The stromal chloroplast Deg7 protease participates in the repair of photosystem II after photoinhibition in Arabidopsis. Plant Physiol. 2010, 152, 1263-1273. [CrossRef]

15. Kato, Y.; Miura, E.; Ido, K.; Ifuku, K.; Sakamoto, W. The variegated mutants lacking chloroplastic FtsHs are defective in D1 degradation and accumulate reactive oxygen species. Plant Physiol. 2009, 151, 1790-1801. [CrossRef] [PubMed]

16. Sun, X.; Peng, L.; Guo, J.; Chi, W.; Ma, J.; Lu, C.; Zhang, L. Formation of DEG5 and DEG8 complexes and their involvement in the degradation of photodamaged photosystem II reaction center D1 protein in Arabidopsis. Plant Cell 2007, 19, $1347-1361$. [CrossRef] [PubMed]

17. Wagner, R.; Aigner, H.; Funk, C. FtsH proteases located in the plant chloroplast. Physiol. Plant. 2012, 145, 203-214. [CrossRef] [PubMed]

18. Sakamoto, W.; Zaltsman, A.; Adam, Z.; Takahashi, Y. Coordinated regulation and complex formation of yellow variegated1 and yellow variegated2, chloroplastic FtsH metalloproteases involved in the repair cycle of photosystem II in Arabidopsis thylakoid membranes. Plant Cell 2003, 15, 2843-2855. [CrossRef]

19. Yu, F.; Park, S.; Rodermel, S.R. The Arabidopsis FtsH metalloprotease gene family: Interchangeability of subunits in chloroplast oligomeric complexes. Plant J. 2004, 37, 864-876. [CrossRef]

20. Zaltsman, A.; Ori, N.; Adam, Z. Two types of FtsH protease subunits are required for chloroplast biogenesis and photosystem II repair in Arabidopsis. Plant Cell 2005, 17, 2782-2790. [CrossRef]

21. Kato, Y.; Hyodo, K.; Sakamoto, W. The photosystem II repair cycle requires FtsH turnover through the EngA GTPase. Plant Physiol. 2018, 178, 596-611. [CrossRef] [PubMed] 
22. Imai, R.; Koizuka, N.; Fujimoto, H.; Hayakawa, T.; Sakai, T.; Imamura, J. Delimitation of the fertility restorer locus Rfk1 to a 43-kb contig in Kosena radish (Raphanus sativus L.). Mol. Genet. Genom. 2003, 269, 388-394. [CrossRef] [PubMed]

23. Dai, C.; Li, Y.; Li, L.; Du, Z.; Lin, S.; Tian, X.; Li, S.; Yang, B.; Yao, W.; Wang, J. An efficient Agrobacterium-mediated transformation method using hypocotyl as explants for Brassica napus. Mol. Breed. 2020, 40, 1-13. [CrossRef]

24. Kato, Y.; Kouso, T.; Sakamoto, W. Variegated tobacco leaves generated by chloroplast FtsH suppression: Implication of FtsH function in the maintenance of thylakoid membranes. Plant Cell Physiol. 2012, 53, 391-404. [CrossRef] [PubMed]

25. Kato, Y.; Sakamoto, W. FtsH protease in the thylakoid membrane: Physiological functions and the regulation of protease activity. Front. Plant Sci. 2018, 9, 855. [CrossRef]

26. Zsiros, O.; Ünnep, R.; Nagy, G.; Almásy, L.; Patai, R.; Székely, N.K.; Kohlbrecher, J.; Garab, G.; Dér, A.; Kovács, L. Role of protein-water interface in the stacking interactions of granum thylakoid membranes-As revealed by the effects of Hofmeister salts. Front. Plant Sci. 2020, 11, 1257. [CrossRef]

27. Smolikova, G.; Laman, N.; Boriskevich, O. Role of chlorophylls and carotenoids in seed tolerance to abiotic stressors. Russ. J. Plant Physiol. 2011, 58, 965-973. [CrossRef]

28. Malnoë, A.; Wang, F.; Girard-Bascou, J.; Wollman, F.-A.; de Vitry, C. Thylakoid FtsH protease contributes to photosystem II and Cytochrome $\mathrm{b}_{6} \mathrm{f}$ remodeling in Chlamydomonas reinhard tii under stress conditions. Plant Cell 2014, 26, 373-390. [CrossRef]

29. Bailey, S.; Thompson, E.; Nixon, P.J.; Horton, P.; Mullineaux, C.W.; Robinson, C.; Mann, N.H. A critical role for the Var2 FtsH homologue of Arabidopsis thaliana in the photosystem II repair cycle in vivo. J. Biol. Chem. 2002, 277, 2006-2011. [CrossRef] [PubMed]

30. Lindahl, M.; Spetea, C.; Hundal, T.; Oppenheim, A.B.; Adam, Z.; Andersson, B. The thylakoid FtsH protease plays a role in the light-induced turnover of the photosystem II D1 protein\{Lindahl, 1983 \#109\}. Plant Cell 1983, 12, 419-431.

31. Nagaharu, U. Genome analysis in Brassica with special reference to the experimental formation of B. napus and peculiar mode of fertilization. Jpn. J. Bot. 1935, 7, 389-452.

32. Chalhoub, B.; Denoeud, F.; Liu, S.; Parkin, I.A.; Tang, H.; Wang, X.; Chiquet, J.; Belcram, H.; Tong, C.; Samans, B. Early allopolyploid evolution in the post-Neolithic Brassica napus oilseed genome. Science 2014, 345, 950-953. [CrossRef]

33. Kato, Y.; Sakamoto, W. Phosphorylation of photosystem II core proteins prevents undesirable cleavage of D 1 and contributes to the fine-tuned repair of photosystem II. Plant J. 2014, 79, 312-321. [CrossRef]

34. Zhuang, K.; Kong, F.; Zhang, S.; Meng, C.; Yang, M.; Liu, Z.; Wang, Y.; Ma, N.; Meng, Q. Whirly1 enhances tolerance to chilling stress in tomato via protection of photosystem II and regulation of starch degradation. New Phytol. 2019, 221, 1998-2012. [CrossRef] [PubMed]

35. Chen, J.H.; Chen, S.T.; He, N.Y.; Wang, Q.L.; Zhao, Y.; Gao, W.; Guo, F.Q. Nuclear-encoded synthesis of the D1 subunit of photosystem II increases photosynthetic efficiency and crop yield. Nat. Plants 2020, 6, 570-580. [CrossRef] [PubMed]

36. Arnon, D.I. Copper enzymes in isolated chloroplasts. Polyphenoloxidase in Beta vulgaris. Plant Physiol. 1949, 24, 1. [CrossRef] [PubMed]

37. Yi, B.; Zeng, F.; Lei, S.; Chen, Y.; Yao, X.; Zhu, Y.; Wen, J.; Shen, J.; Ma, C.; Tu, J. Two duplicate CYP704B1-homologous genes BnMs1 and BnMs2 are required for pollen exine formation and tapetal development in Brassica napus. Plant J. 2010, 63, 925-938. [CrossRef]

38. Bolger, A.M.; Lohse, M.; Usadel, B. Trimmomatic: A flexible trimmer for Illumina sequence data. Bioinformatics 2014, 30, 2114-2120. [CrossRef]

39. Thorvaldsdóttir, H.; Robinson, J.T.; Mesirov, J.P. Integrative Genomics Viewer (IGV): High-performance genomics data visualization and exploration. Brief. Bioinform. 2013, 14, 178-192. [CrossRef] [PubMed]

40. Takagi, H.; Abe, A.; Yoshida, K.; Kosugi, S.; Natsume, S.; Mitsuoka, C.; Uemura, A.; Utsushi, H.; Tamiru, M.; Takuno, S.; et al. QTL-seq: Rapid mapping of quantitative trait loci in rice by whole genome resequencing of DNA from two bulked populations. Plant J. Cell Mol. Biol. 2013, 74, 174-183. [CrossRef] [PubMed]

41. Abe, A.; Kosugi, S.; Yoshida, K.; Natsume, S.; Takagi, H.; Kanzaki, H.; Matsumura, H.; Yoshida, K.; Mitsuoka, C.; Tamiru, M. Genome sequencing reveals agronomically important loci in rice using MutMap. Nat. Biotechnol. 2012, 30, 174-178. [CrossRef] [PubMed]

42. Song, J.M.; Guan, Z.; Hu, J.; Guo, C.; Guo, L. Eight high-quality genomes reveal pan-genome architecture and ecotype differentiation of Brassica napus. Nat. Plants 2020, 6, 1-12. [CrossRef]

43. Nagarajan, S.; Steephen, M.; Murugan, K.; Nair, R.R.; Ganesh, D. Improved protocol for isolation of genomic DNA from leaf tissues of Phyllanthus emblica Gaertn. Iran. J. Biotechnol. 2011, 9, 307-313.

44. Cheng, F.; Liu, S.; Wu, J.; Fang, L.; Sun, S.; Liu, B.; Li, P.; Hua, W.; Wang, X. BRAD, the genetics and genomics database for Brassica plants. BMC Plant Biol. 2011, 11, 1-6. [CrossRef] [PubMed]

45. Xia, S.; Wang, Z.; Zhang, H.; Hu, K.; Zhang, Z.; Qin, M.; Dun, X.; Yi, B.; Wen, J.; Ma, C.; et al. Altered transcription and neofunctionalization of duplicated genes rescue the harmful effects of a chimeric gene in Brassica napus. Plant Cell 2016, 28, 2060-2078. [CrossRef] [PubMed]

46. Yang, H.; Liu, J.; Huang, S.; Guo, T.; Deng, L.; Hua, W. Selection and evaluation of novel reference genes for quantitative reverse transcription PCR (qRT-PCR) based on genome and transcriptome data in Brassica napus L. Gene 2014, 538, 113-122. [CrossRef] [PubMed] 
47. Hu, B.; Jin, J.; Guo, A.-Y.; Zhang, H.; Luo, J.; Gao, G. GSDS 2.0: An upgraded gene feature visualization server. Bioinformatics 2014, 31, 1296-1297. [CrossRef] [PubMed]

48. Yoo, S.-D.; Cho, Y.-H.; Sheen, J. Arabidopsis mesophyll protoplasts: A versatile cell system for transient gene expression analysis. Nat. Protoc. 2007, 2, 1565. [CrossRef]

49. Gookin, T.E.; Assmann, S.M. Significant reduction of BiFC non-specific assembly facilitates in planta assessment of heterotrimeric G-protein interactors. Plant J. 2014, 80, 553-567. [CrossRef] [PubMed]

50. Clough, S.J.; Bent, A.F. Floral dip: A simplified method for Agrobacterium-mediated transformation of Arabidopsis thaliana. Plant J. 1998, 16, 735-743. [CrossRef] [PubMed]

51. Jefferson, R.A.; Kavanagh, T.A.; Bevan, M.W. GUS fusions: Beta-glucuronidase as a sensitive and versatile gene fusion marker in higher plants. EMBO J. 1987, 6, 3901-3907. [CrossRef] [PubMed]

52. Kumar, S.; Stecher, G.; Li, M.; Knyaz, C.; Tamura, K. MEGA X: Molecular evolutionary genetics analysis across computing platforms. Mol. Biol. Evol. 2018, 35, 1547-1549. [CrossRef] [PubMed]

53. Edgar, R.C. MUSCLE: Multiple sequence alignment with high accuracy and high throughput. Nucleic Acids Res. 2004, 32, 1792-1797. [CrossRef] [PubMed]

54. Yang, Z. PAML: A program package for phylogenetic analysis by maximum likelihood. Bioinformatics 1997, 13, 555-556. [CrossRef]

55. Jones, D.T.; Taylor, W.R.; Thornton, J.M. The rapid generation of mutation data matrices from protein sequences. Bioinformatics 1992, 8, 275-282. [CrossRef] [PubMed]

56. Efron, B. The Jackknife, the Bootstrap and Other Resampling Plans; SIAM: Philadelphia, PA, USA, 1982.

57. Felsenstein, J. Confidence limits on phylogenies: An approach using the bootstrap. Evolution 1985, 39, 783-791. [CrossRef] [PubMed] 